\title{
Estudo dos Estágios Motivacionais em Sujeitos Adultos Dependentes do Álcool
}

\author{
Margareth da Silva Oliveira ${ }^{1}$ \\ Pontifícia Universidade Católica do Rio Grande do Sul \\ Ronaldo Laranjeira \\ Escola Paulista de Medicina \\ Renata Brasil Araujo \\ Rafael Leal Camilo \\ Pontifícia Universidade Católica do Rio Grande do Sul \\ Daniela DiGiórgio Schneider \\ Universidade Federal do Rio Grande do Sul
}

\begin{abstract}
Resumo
Esta pesquisa tem por objetivo identificar a configuração dos estágios motivacionais em alcoolistas internados em unidades especializadas e verificar se há uma correlação entre estes estágios e o grau de dependência avaliado. A amostra foi composta por 120 alcoolistas, do sexo masculino com nível de dependência entre moderado e grave. Os instrumentos utilizados foram: Shortform Alcohol Dependence Data (SADD) para identificar a gravidade da dependência alcóolica e a University of Rhode Island Change Assessment Questionnaire (URICA) para avaliar os estágios motivacionais. Os resultados demonstraram uma correlação positiva e de intensidade baixa $(r=0,197 ; p=0,031)$ entre a gravidade da dependência e o estágio de Manutenção, bem como uma preponderância de escores neste estágio. Discute-se a importância da aplicação das escalas utilizadas para um melhor delineamento das técnicas a serem adotadas durante o processo terapêutico.

Palavras-chave: Dependência do álcool; estágios motivacionais; grau de dependência; escalas.
\end{abstract}

Study of Motivational Stages in Alcohol Dependent Adult Subjects

\begin{abstract}
The general aim of this work was to trace the motivational stages of interned clients in specialized units, based on Prochaska and Diclemente's Transtheorical Model. This work also examines if there is a correlation between the motivation stages of this group of alcohol-addicted patients and their level of drug dependence. The sample was composed of 120 alcoholics male patients, with dependence levels between moderate and critical. The instruments used were: Short-form Alcohol Dependence Data to identify the dependence level and the University of Rhode Island Change Assessment Questionnaire to evaluate the motivational stages. The results show positive and low-intensity correlation $(r=0,197 ; p=0,031)$ between the level of drug dependence and the Maintenance stage. It is still under discussion the importance of the scales used in order discussion, in order to judge and choose better techniques to be adopted during the therapeutic process.

Keywords: Alcohol dependence; motivational stages; dependence level; scales.
\end{abstract}

O alcoolismo constitui-se em um grave problema de saúde pública, sendo que cerca de $10 \%$ da população brasileira enfrenta sérios problemas relacionados ao uso excessivo de álcool, isto é, são dependentes desta substância psicoativa (Laranjeira \& Pinsky, 1997). O álcool é uma das drogas mais utilizadas no país, chegando a ser consumida por mais de $70 \%$ dos adultos. Além disso, é o principal responsável pelos acidentes de maior gravidade e pelas mortes mais violentas (Ferreira \& Laranjeira, 1998).

Apesar dos inúmeros prejuízos conseqüentes do abuso de álcool, os clientes com tal disfunção apresentam dificuldades em se manter por um longo período em tratamento psicoterápico (Edwards \& Dare, 1997). O

\footnotetext{
${ }^{1}$ Endereço para correspondência: Av. Ipiranga, 6681, prédio 11, $9^{\circ}$ andar, 90619 900, Porto Alegre, RS. Fone/ Fax 33203633 (r: 217). E-mail: marga@pucrs.br
}

abandono dos programas de tratamento do alcoolismo sugere que dois terços dos pacientes terminam o processo de recuperação antes da décima sessão, com o número médio de quatro sessões (Stark, 1992). Isto parece estar vinculado a um evidente conflito motivacional, experenciado por esta clientela, com relação à modificação de seu comportamento problema (Heather, 1992).

Com o intuito de compreender como as pessoas mudam foi desenvolvido o Modelo Transteórico de Prochaska e DiClemente (1982) que descreve a prontidão para a mudar como estágios de mudança pelos quais o indivíduo transita. Este modelo está baseado na premissa que a mudança comportamental é um processo, e que as pessoas tem diversos níveis de motivação, de prontidão para mudar.

Prochaska e DiClemente (1992), ao desenvolverem um instrumento para identificar os estágios de mudança, quando os clientes iniciam um tratamento, chegaram a quatro estágios 
bem definidos, confiáveis e bem relacionados entre si: Précontemplação, Contemplação, Ação e Manutenção.

A Pré-contemplação é um estágio em que não há intenção de mudança nem mesmo uma crítica a respeito do conflito envolvendo o comportamento-problema; a Contemplação se caracteriza pela conscientização de que existe um problema, no entanto há uma ambivalência quanto à perspectiva de mudança; a Ação se dá quando o cliente escolhe uma estratégia para a realização desta mudança e toma uma atitude neste sentido e a Manutenção é o estágio onde se trabalha a prevenção à recaída e a consolidação dos ganhos obtidos durante a Ação.

É preciso salientar que nem sempre um cliente que busca recurso encontra-se no estágio de Ação. Uma pessoa que interna-se em uma unidade para o tratamento da dependência do álcool, mas não se engaja no programa da instituição, não reconhece os problemas oriundos do abuso desta substância ou mostra-se ambivalente quanto a manter ou interromper o uso, pode estar mostrando evidências de que encontra-se em algum outro estágio.

Tradicionalmente, Manutenção é vista como um estágio estático, porém, trata-se de um estágio dinâmico, pois entende-se como a continuação do novo comportamento para a mudança que demora algum tempo para se estabelecer (Jungerman \& Laranjeira, 1999). O estágio de manutenção pode e deve ser estimulado por toda a vida. Aqui o foco do trabalho é manter os ganhos do tratamento evitando a recaída. Trata-se de uma fase difícil, mas crucial no tratamento de qualquer dependência química. Não é tão difícil interromper o comportamento de uso quanto é manter a abstinência.

De acordo com Prochaska, DiClemente e Norcross (1999) o indivíduo não caminha nos estágios de forma linearcausal (em que há uma causa, um efeito e conseqüente alteração no comportamento dependente) como anteriormente se pensava. As mudanças de estágios são melhores representadas por uma espiral, em que as pessoas podem progredir ou regredir sem ordenação lógica.

DiClemente (1999) explana sobre as vantagens de se entender o comportamento humano a partir do Modelo Transteórico, por este ser um construto integrativo no tratamento e prevenção das adições. $\mathrm{O}$ autor considera que o conceito de mudança neste construto teórico,abarca uma perspectiva desenvolvimental e que uma perspectiva de mudança ajuda a evitar explanações estáticas sobre o que parece ser um ativo processo de adição e recuperação.

$\mathrm{O}$ modelo tem sido estudado em uma série de populações com diferentes tipos de comportamentos e mostra capacidade de integração com outras teorias. Prochaska e colaboradores (1994) realizaram um amplo estudo envolvendo 12 comportamentos-problema e sua relação com balança decisional do Modelo de Tomada de Decisão de Janis e Mann (1977). Os resultados mostraram que, em todos eles, observaram-se mais desvantagens do que vantagens relacionadas a mudança de comportamento entre os pré -contempladores. Já entre os contempladores, observaram-se mais vantagens do que desvantagens referentes à mudança de comportamento em onze dos doze comportamentos. Outro dado interessante refere-se a uma menor incidência de desvantagens em mudar o comportamento entre os alcoolistas no estágio de Ação do que naqueles em Contemplação, mesmo quando se avaliou os doze comportamentos estudados. A partir destes dados, os autores argumentam que o processo de mudança pode ser empiricamente integrado com os estágios de mudança.

Velasquez, Carbonari e DiClemente (1999) analisaram o Modelo Transteórico e sua relação com processos de mudança, balança decisional, auto-eficácia e "tentação" em uma população de 132 alcoolistas com diagnóstico duplo (transtorno mental crônico e dependência alcóolica) e concluíram que quanto mais o indivíduo experiencia problemas com o álcool, mais torna-se disposto a beber, particularmente, em situações de afeto negativo. Além disso, os alcoolistas com mais problemas psiquiátricos, pontuaram mais no estágio de Manutenção.

Rumpf, Hapke e John (1998), investigaram a relação entre os estágios de mudança e procura de ajuda prévia em alcoolistas admitidos em um hospital geral de Lübeck, Alemanha. Os resultados mostram que 10,9\% estavam no estágio de Pré-contemplação e 84,8\% estavam nos estágios de Contemplação ou Ação, o que contrasta com a opinião dos clínicos de que esta população pode não estar motivada para mudar o comportamento de uso. A partir destes dados, demonstram a importância de se intervir, mesmo em um hospital geral, junto a esta população, considerando o estágio motivacional em que os indivíduos se encontram.

De acordo com Edens e Willoughby (2000) pacientes dependentes de álcool, os quais demonstraram atitudes mais consistentes com os estágios de Contemplação e Ação, eram significativamente mais prováveis de completarem o programa de tratamento para abuso de substância, do que aqueles que demonstraram, proporcionalmente, menos destas atitudes. Em alguns outros trabalhos foram utilizados o University of Rhode Island Change Assessment Questionnaire (URICA-McConnaughy, Prochaska \& Velicer, 1983) e outras escalas para analisar se havia uma associação entre os estágios motivacionais e a gravidade da dependência do álcool, obtendo-se como resultado, a constatação de que os alcoolistas considerados "graves" demonstraram uma maior motivação para mudança. 
Assim, para Prochaska e DiClemente (1992) avaliar a motivação para mudança, independentemente do tratamento utilizado, parece ser um aspecto importante para a utilização de intervenções adequadas para os pacientes.

A medida em que se tem contato com esta teoria, percebe-se que avaliar os estágios motivacionais e promover um tratamento individualizado, tendo como meta a motivação para mudança, são caminhos essenciais para que o profissional que trabalha com dependentes químicos possa conseguir resultados consistentes.

Esta pesquisa tem por objetivo identificar os estágios motivacionais (Prochaska \& DiClemente, 1982) em alcoolistas que estão iniciando um tratamento em unidades especializadas na recuperação da dependência química e verificar se há uma correlação entre estes estágios e a gravidade da dependência alcóolica deste grupo. Pretende, da mesma forma, discutir a importância da aplicação de uma escala que avalie os estágios motivacionais e de uma escala para identificar a gravidade da dependência alcóolica, para um melhor delineamento das técnicas a serem adotadas durante o processo terapêutico. Uma das limitações desta pesquisa, é importante destacar, é o fato da Escala URICA (McConnaughy \& cols., 1983) ainda estar em processo de validação no Brasil (Figlie, 1999), porém sua utilidade na clínica e sua valorização pelos profissionais da área da dependência química justificam sua utilização neste estudo.

Com novas pesquisas nesta área, torna-se possível que tenhamos, cada vez mais, um maior arsenal terapêutico para que possamos tratar o nosso cliente, dependente químico, de uma forma mais efetiva, respeitando, acima de tudo, a sua individualidade, o seu desejo e a sua prontidão à mudança.

\section{Método}

\section{Delineamento}

Trata-se de um estudo analítico, transversal, do tipo quantitativo.

\section{Participantes}

Participaram do estudo 120 alcoolistas, todos do sexo masculino, compreendidos na faixa etária entre 20 e 65 anos $(m=40$ anos, $d p=7,51$; Idade mínima=26; Idade máxima $=59)$.

A escolaridade mínima exigida para fazer parte da amostra era 5. ${ }^{a}$ série do $1^{\circ} \mathrm{Grau}$. Os sujeitos preencheram os critérios para Síndrome de Dependência do Álcool do CID-10 (Organização Mundial de Saúde, 1993) e estavam internados em hospitais especializados em dependência química, em Porto Alegre, com o objetivo de desintoxicação e tratamento breve .
Os critérios de exclusão da amostra foram a presença de transtornos orgânicos cerebrais, transtornos psiquiátricos severos, tais como esquizofrenia, e dependência de outras substâncias psicoativas, com exceção da nicotina.

\section{Instrumentos}

Foram aplicados os seguintes instrumentos: Short-form Alcohol Dependence Data (SADD) criada por Raistrick, Dunbor e Davidson (1983), para avaliar o grau de dependência alcoólica, e a Escala University of Rhode Island Change Assessment Questionnaire (URICA- McConnaughy \& cols., 1983), para avaliar os estágios motivacionais da amostra analisada.

A SADD (Raistrick \& cols., 1983) foi padronizada para uso no Brasil por Jorge e Masur (1986). Trata-se de uma escala auto aplicável, constituída por 15 itens relacionados ao consumo do álcool, que objetiva avaliar o grau de dependência desta substância. Há quatro alternativas de respostas para cada questão: $0=$ Nunca; $1=$ Poucas vezes; $2=$ Muitas vezes; e $3=$ Sempre. De acordo com a soma total de pontos, os alcoolistas são classificados nas seguintes categorias: 1 a 9=Dependência leve; 10 a 19=Dependência moderada; e 20 a 45=Dependência grave.

A gravidade da dependência alcoólica é uma dimensão necessária em todas as avaliações principalmente para direcionar o enfoque do tratamento, porém não é fácil estabelecer regras absolutas para a graduação da severidade desta dependência. Segundo Edwards, Marshal e Cook (1999), "quando uma pessoa experiencia sintomas de abstinência diariamente por 6 a 12 meses, e bebe para obter alívio a esses sintomas durante o mesmo período, esta pessoa estará gravemente dependente do álcool. Quanto mais um indivíduo tiver repetidos ciclos de abstinência e alívio, mais grave será sua dependência” (p. 49).

A URICA (McConnaughy \& cols., 1983), por sua vez, é uma escala que busca avaliar os estágios motivacionais de indivíduos e o quanto estes estão disponíveis para uma mudança em seu comportamento-problema. Não está associada unicamente ao uso de substâncias psicoativas, mas a qualquer problema que seria importante modificar. Assim, na área da dependência química, esta escala pode ser aplicada tanto para alcoolistas quanto para dependentes de outras substâncias. O questionário tem 32 afirmações para as quais poderá se escolher as seguintes respostas: $1=$ Discorda totalmente; $2=$ Discorda; $3=$ Indeciso; 4=Concorda; $5=$ Concorda totalmente. A partir do levantamento dos dados, poderá ser avaliada a distribuição entre os estágios motivacionais (Pré-contemplação, Contemplação, Ação e Manutenção) em cada sujeito, à medida em que cada fase está relacionada a 8 itens. Desta forma, o resultado final explicitará quantos pontos se fez em cada uma das fases, 
bem como se há um predomínio significativo de alguma delas. No Brasil, estudos de padronização e análise fatorial foram realizados por Figlie (2001), demonstrando a confiabilidade deste instrumento.

McConnaughy e colaboradores (1983) proveram um forte suporte para a confiança e validade da URICA, relatando que a mesma possui propriedades psicométricas aceitáveis. Além disso, várias análises, Norman, Conner e Bell (1999) que têm utilizado esta escala em populações de fumantes e usuários de substâncias psicoativas, têm identificado subgrupos homogêneos que são consistentes com o Modelo Transteórico.

\section{Procedimentos}

Inicialmente foi realizado contato com duas instituições especializadas no atendimento de pacientes dependentes químicos para realizarmos a presente pesquisa. Após contatos prévios com as direções e encaminhamento do projeto para as comissões de ética das respectivas instituições, passamos para a coleta dos dados.

Todos os alcoolistas que ingressaram na pesquisa entravam em contato com a proposta de trabalho e após a leitura de uma carta informativa sobre o projeto decidiam por participar ou não da pesquisa. Os que optavam por integrar a proposta assinavam o termo de consentimento informado como comprovação de sua participação intencional no estudo.

A aplicação dos instrumentos, realizada entre o terceiro e sétimo dia da internação, fora individual e o avaliador anotava todas as respostas do sujeito em um protocolo de respostas. Era fornecida uma cópia de cada instrumento para que ele pudesse seguir a leitura dos itens e facilitar a concentração nos mesmos. Após a aplicação dos instrumentos e posterior levantamento dos dados, foi realizada uma entrevista de devolução dos resultados de cada sujeito.

Cabe salientar que este trabalho faz parte de um amplo estudo que buscou avaliar, de forma abrangente, a eficácia da Intervenção Motivacional em alcoolistas (Oliveira, 2000).

\section{Resultados}

\section{Sociodemográficos}

Com relação ao estado civil, 46,7 \% dos alcoolistas pesquisados eram casados $(n=56), 33,3 \%$ separados $(n=40)$ e $20 \%$ solteiros $(n=24)$.

Quanto à escolaridade: $29,2 \%$ dos sujeitos tinham o ensino fundamental incompleto $(n=35) ; 28,3 \%$ o ensino fundamental completo $(n=34) ; 10 \%$ o ensino médio incompleto $(n=12) ; 19,2 \%$ o ensino médio completo $(n=23)$; $7,5 \%$ o ensino superior incompleto $(n=9)$ e $5,8 \%$ o ensino superior completo $(n=7)$.

\section{Grau de dependência alcoólica}

Através dos resultados da SADD, a distribuição dos alcoolistas da amostra $(n=120)$, quanto à gravidade da dependência alcoólica, apresentou-se da seguinte forma: $73,3 \%(n=88)$ tinham um grau de dependência grave e $26,7 \%(n=32)$ moderado.

\section{Perfil motivacional da amostra}

Através da aplicação da Escala URICA, foi possível descrever a seguinte distribuição quanto aos estágios motivacionais, pela média da pontuação de cada estágio: Pré-contemplação: $m=15,84$; $d p=5,42$; Contemplação: $m=30,24 ; d p=3,31$; Ação: $m=31,25 ; d p=3,28$ e Manutenção: $m=28,47 ; d p=4,00$.

\section{Grau de dependência alcoólica e os estágios motivacionais}

Foram realizadas análises estatísticas nos dados oferecidos pelas Escalas SADD e URICA, sendo utilizado inicialmente o coeficiente de correlação de Spearmann, o qual demonstrou haver uma correlação positiva, de intensidade baixa $\left(r_{\mathrm{s}}=0,197 ; p=0,031\right)$ entre o estágio de Manutenção da URICA e uma maior gravidade da dependência na SADD. O mesmo não acontecendo com os demais estágios: Pré-contemplação $(r=-0,172 ; p=0,060)$, Contemplação $(r=0,098 ; p=0,289)$ e Ação $(r=0,118$; $p=0,031)$.

Foi aplicado o teste Mann-Whitney para comparar a pontuação da escala URICA entre os grupos Grave e Moderado da SADD separadamente. Segundo os resultados, os estágios Pré-contemplação $(p=0,113)$, Contemplação $(p=0,789)$ e Ação $(p=0,263)$ não apresentaram uma associação com o grau de dependência grave, isto ocorrendo apenas com o estágio de Manutenção $(p=0,039)$.

\section{Discussão}

A aplicação da URICA nesta amostra apresentou resultados interessantes nos quais há uma distribuição sem grandes disparidades entre as médias de três estágios motivacionais, sendo a menor média encontrada na Pré contemplação. Este achado está de acordo com o que foi descrito por Rumpf e colaboradores (1998) em uma pesquisa com alcoolistas internados em Hospital Geral.

Também demonstra o que Prochaska e colaboradores (1999) referem a respeito da motivação como esta não sendo 
algo estanque, que segue um curso linear, mas sim um "estado" pelo qual o indivíduo "transita", demonstrando, simultaneamente aspectos ligados a todas as fases, não sendo necessário que uma prevaleça sobre as outras. Este conhecimento é de suma importância para quem trabalha na área da dependência química, pois não subdivide os alcoolistas em dois grupos: os motivados e os desmotivados, mas sim compreende a motivação como um processo, devendo, os terapeutas agir para que os indivíduos queiram modificar o seu comportamento-problema.

$\mathrm{Na}$ amostra estudada, a partir da correlação positiva entre o estágio de Manutenção da URICA e uma maior gravidade da dependência do álcool pela SADD, pode-se inferir que aqueles indivíduos com dependência alcóolica mais grave, por experenciarem maiores prejuízos, tanto clínicos, quanto psicossociais, apresentam uma maior prontidão à mudança, o que está de acordo com algumas pesquisas realizadas (Carpenter, Miele \& Hasin, 2002; Edens \& Willoughby, 2000; Steinberg, 2000). No entanto, os dados obtidos por Velasquez e colaboradores (1999) em sua pesquisa com alcoolistas que apresentavam comorbidades psiquiátricas, estimula que se fique atento, pois ter uma maior motivação para mudança não significa que $o$ indivíduo seja "imune" a situações de risco e, consequentemente, à recaída.

Constatar esta correlação entre motivação e gravidade da dependência do álcool é de fundamental importância, pois contrasta com o preconceito de diversos profissionais da área da saúde e dos próprios alcoolistas, que consideram quem tem um grau mais severo de dependência como mais difícil de ser tratado e com um pior prognóstico, o que, muitas vezes, interfere no investimento, tanto destes técnicos quanto desta grupo específico, em um tratamento mais efetivo.

Assim, incrementar as estratégias de Prevenção de Recaída (Marlatt \& Gordon, 1993) deste grupo de indivíduos (com dependência grave), trabalhando a sua auto-eficácia, pode ser uma técnica de tratamento muito valiosa para o profissional que o atende, pois desta forma, não se estará subestimando a sua prontidão à mudança, bem como estará reforçando os seus aspectos ligados ao estágio de Manutenção.

Além disto, conclui-se que a aplicação de escalas, como a URICA (McConnaughy \& cols., 1983), para avaliar a prontidão à mudança de clientes dependentes químicos e a SADD (Raistrick \& cols., 1983), para verificar a gravidade da dependência pode ser um importante adjuvante no processo de avaliação, feedback e posterior delineamento das estratégias a serem utilizadas no decorrer do tratamento.

Assim, quanto mais aprendermos a respeito dos estágios motivacionais, e da dependência química em si, melhor capacitados estaremos para o tratamento de nossos clientes, o que, sem dúvida alguma, irá interferir na nossa própria motivação, e na nossa disponibilidade para estarmos em um constante e necessário processo de mudança.

\section{Referências}

Carpenter, K., Miele, G. M. \& Hasin, D. S. (2002). Does motivation to change mediate the effect of DSM-IV substance use disorders on treatment utilization and substance use? Addictive-Behaviors, 27, 207225.

DiClemente, C. C. (1999). Prevention and harm reduction for chemical dependency: A process perspective. Clinical Psycology Review, 19, 473486.

Edens, J. \& Willoughby, F. (2000). Motivational patterns of alcohol dependent patients: A replication. Psycology of Addictive Behaviors, 14, 397- 400 .

Edwards, G. \& Dare, C. (1997). Psicoterapia e tratamento de adições. São Paulo, SP: Artes Médicas.

Edwards, G., Marshal, E. J. \& Cook, C. H. (1999). O tratamento do alcoolismo: Um guia para profissionais da saúde. Porto Alegre, RS: Artes Médicas.

Ferreira, M. P. \& Laranjeira, R. (1998). Dependência de substâncias psicoativas. Em L. Ito \& cols. (Orgs.), Terapia cognitivo-comportamental para transtornos psiquiátricos (pp. 105-121). Porto Alegre, RS: Artes Médicas.

Figlie, N. B. (1999). Motivação em alcoolistas tratados em ambulatório específico para alcoolismo e em ambulatório de gastroenterologia. Dissertação de Mestrado nãopublicada, Programa de Pós-graduação da Escola Paulista de Medicina, Universidade Federal de São Paulo. São Paulo, SP.

Figlie, N. (2001, Setembro). Metodologia de pesquisa em dependência química. Trabalho apresentado no XIV Congresso Brasileiro sobre alcoolismo, tabagismo e outras dependências, Gramado, RS.

Heather, N. (1992). Addictive disorders are essentially motivational problems. British Journal of Addiction, 87, 827-830.

Janis, I. L. \& Mann, L. (1977). Decision making: A psychological analysis of conlict, choice, and commitment. London: Cassel \& Cllier Macmillan.

Jorge, M. R. \& Masur, J. (1986). Questionários padronizados para avaliação do grau de severidade da síndrome de Dependência do Álcool. Jornal Brasileiro de Psiquiatria, 35, 287-292.

Jungerman, F. S. \& Laranjeira, R. (1999). Entrevista motivacional: Bases teóricas e práticas. Jornal Brasileiro de Psiquiatria, 48, 197-207.

Laranjeira, R. \& Pinsky, I. (1997). O alcoolismo. São Paulo: Contexto.

Marlatt, A. \& Gordon, J. (1993). Prevenção da recaída: Estratégia e manutenção no tratamento de comportamentos aditivos. Porto Alegre, RS: Artes Médicas.

McConnaughy, E. A., Prochaska, J. O. \& Velicer, W. F. (1983). Stages of change in psychoterapy: Measurement and sample profiles. Psychoterapy: Theory, Research \& Practice, 20, 368-375.

Norman, P., Conner, M. \& Bell, R. (1999). The theory of planned behaviors and smoking cessation. Health Psychology, 18, 89-94.

Oliveira, M. (2000). Eficácia da intervenção motivacional em dependentres do álcool. Tese de Doutorado não-publicada, Curso de Psiquiatria e Psicologia Médica, Universidade Federal de São Paulo. São Paulo, SP.

Organização Mundial de Saúde (1993). Classificação dos Transtornos Mentais e de Comportamento: Descrições clínicas e diretrizes diagnósticas. Porto Alegre, RS: Artes Medicas.

Prochaska, J. O. \& DiClemente, C. (1982). Transtheorical therapy: Toward a more integrative model of change. Psycotherapy: Theory, Research and Practice, $20,161-173$. 
Prochaska, J. O. \& DiClemente, C. (1992). Stages of change in the modi cafication of problem behaviors. Em M. Hersen, M. Eiser \& W. Miller (Orgs.), Progress in behavior modification (pp.184-214). Sycamore: Sycamore Press.

Prochaska, J. O., DiClemente, C. C. \& Norcross, J. C. (1992). Search of how people change: Applications to addictive behaviors. American Psychologist, 47, 1102-1113.

Prochaska, J. O., Velicer, W. Y., Rossi, J. S., Goldstein, B. M., Rakowski, W., Fiore, C., Harlow, L., Redding, C. A ., Resembloom, D. \& Rossi, S. R. (1994). Stages of change and decisional balance for 12 problem behaviors. Health Psychology, 13, 39-46.

Raistrick, D., Dunbor, G. \& Davidson, R. (1983). Development of a questionnaire to measure alcohol dependence. Britsh Journal of Addiction, 78, 8995.

Rumpf, H., Hapke, U. \& John, U. (1998). Previous help seeking and motivation to change drinking behaviour in alcohol-dependent General hospital patients. General Hospital Psychiatry, 20, 115-119.
Stark, M. (1992). Dropping out of substance abuse treatment: A clinically oriented review. Clinical Psychology Review, 12, 93-116.

Steinberg, K. M. (2000). Predictors of dropout in the treatment of alcohol and cocaine dependence: Tests of the stages of change. The Sciences and Engineering, 60(12-B), 6384.

Velasquez, M. M., Carbonari, J. P. \& DiClemente, C. (1999). The relation of the transtheoretical model variables to psychiatric distress in dually diagnosed patients. Addictive Behaviors, 24, 481-496.

Sobre os autores

Margareth da Silva Oliveira é Doutora em Ciências pela Universidade Federal de São Paulo. É Professora do Programa de Pós-graduação em Psicologia da Pontifícia Universidade Católica do Rio Grande do Sul.

Ronaldo Laranjeira é Doutor em Psiquiatria pela University of London Faculty of Medicine. É Professor da Universidade Federal de São Paulo.

Renata Brasil Araujo é Doutoranda em Psicologia pela Pontifícia Universidade Católica do Rio Grande do Sul.

Daniela DiGiorgio Schneider é Mestranda em Psicologia do Desenvolvimento pela Universidade Federal do Rio Grande do Sul.

Rafael Leal Camilo é Bolsista de iniciação científica da Pontifícia Universidade Católica do Rio Grande do Sul. 